Article

\title{
Hyperbolic Equations with Unknown Coefficients
}

\author{
Aleksandr I. Kozhanov \\ Sobolev Institute of Mathematics, Acad. Koptyug av. 4, 630090 Novosibirsk, Russia; kozhanov@math.nsc.ru \\ Received: 14 July 2020; Accepted: 1 September 2020; Published: 17 September 2020 \\ check for \\ updates
}

\begin{abstract}
We study the solvability of nonlinear inverse problems of determining the low order coefficients in the second order hyperbolic equation. The overdetermination condition is specified as an integral condition with final data. Existence and uniqueness theorems for regular solutions are proved (i.e., for solutions having all weak derivatives in the sense of Sobolev, occuring in the equation).

Keywords: hyperbolic equation; inverse problem; unknown coefficient; final-integral overdetermination condition; regular solution; existence; uniqueness
\end{abstract}

MSC: 35R30; 35M20

\section{Introduction}

The article is devoted to the study of the solvability of some new nonlinear inverse coefficient problems for partial differential equations.

Inverse coefficient problems for differential equations are problems in which, together with the solution, it is also required to determine the equation itself, that is, unknown coefficients of the equation or some unknown components of its right-hand side.

It is well known that for the solvability of inverse coefficient problems, alongside natural boundary information generating a well-posed boundary value problem for the corresponding class of differential equations, some additional conditions are required. These conditions are conditions of structural kind and overdetermination conditions.

Among conditions pertaining to structural kind, one can distinguish two main cases. In the first of them, it is assumed that the unknown coefficient does not depend on the time (distinguished) variable, i.e., in fact it depends on the space variables (see, for example, [1-9] and many other works); we refer to such inverse problems as space-type inverse problems. In the second case, it is, on the contrary, assumed that the unknown coefficient depends only on the time (distinguished) variable (see [1,10-17]) and we will refer to such inverse problems as time-type inverse problems.

In some investigations, which are not many in contrast to space, and time-type inverse problems it is assumed that the unknown coefficient depends on all independent variables but in a special way (namely, it depends on some a priori defined combination of the independent variables), see [3,6,18-20].

The problems studied in the present article differ from many that have been investigated before as the unknown coefficients in them are constants (i.e., numbers) and not functions. This structural condition means that the solvability conditions must be substantially different from those in space- or time-type inverse problems.

The study of the solvabilitty of inverse problems for partial differential equations with unknown constant coefficients began comparatively recently.

The works [21-27] were devoted to the solvability of inverse problems with unknown constant coefficients for parabolic equations. In [21], a special linear final-integral overdetermination condition was used, in [22-25] quadratic final-integral overdetermination conditions were utilized, and the works [21-25] used the semigroup approach. In [26,27], linear final-integral overdetermination conditions were used and the regularization and fixed point methods were applied. 
Note also the article [28], in which the solvability was studied of inverse problems of finding, together with the solution of elliptic equations, the unknown constant defining the boundary regime.

As for inverse problems with unknown constant coefficients for hyperbolic equations, we can only mention [29], where the inverse problem was studied for finding the lower coefficient in a telegraph equation, and here the quadratic final-integral overdetermination condition was used.

In the present article, we will study the solvability of inverse problems with constant coefficients for hyperbolic equations in the case of a linear final-integral overdetermination condition. As said above, such problems have not been studied before.

An additional observations relates to problems in which some processes are described by differential equations with constant coefficients appearing to be natural for a homogeneous medium see for example, [30]. Furthermore, if some characteristics of the medium are unknown a priori then the mathematical modeling of the corresponding process inevitably leads to the necessity of investigating the solvability of inverse problems for differential equations with unknown constant coefficients.

In Section 2, we give the statement of the problems under study. In Section 3, we prove existence theorems for solutions. Section 4 is devoted to discussing the uniqueness of th solutions. Finally, in Section 5, we give some comments to the obtained results, propose possible generalizations and extensions of the results, as well as give examples.

\section{Statement of the Problem}

Throughout the article, we will use the usual Lebesgue spaces $L_{p}$, the Sobolev spaces $W_{p}^{l}$, and also the spaces $L_{p}(0, T ; X)$. The definitions and properties of these spaces can be found in $[31,32]$.

Let $\Omega$ be a bounded domain in $\mathbb{R}^{n}$ with smooth (for simplicity, infinitely-differentiable) boundary $\Gamma, Q$ be the cylinder $\{(x, t): x \in \Omega, t \in(0, T)\}$ of finite height $T$, and $S$ be the lateral boundary $Q: S=\Gamma \times(0, T)$. Everywhere below, $\alpha^{i j}(x), i, j=1, \ldots, n, \alpha^{0}(x), f(x, t), R(x), u_{0}(x)$, and $u_{1}(x)$ are given functions defined for $x \in \bar{\Omega}, t \in[0, T]$. Furthermore, let $A$ be a differential operator acting at a given function $v(x)$ by the equality:

$$
A v=\frac{\partial}{\partial x_{i}}\left(\alpha^{i j}(x) v_{x_{j}}\right)+\alpha^{0}(x) v
$$

(here and below, repeated indices presume summation from 1 to $n$ ), $R_{0}, a$, and $b$ are real numbers, $R_{0}$ and one of the numbers $a$ and $b$ are assumed predefined.

Inverse Problem I: Find a function $u(x, t)$ and a number a that satisfy the equation:

$$
u_{t t}-A u+a u_{t}+b u=f(x, t)
$$

in the cylinder (here the number $b$ is assumed predefined) and $u(x, t)$ satisfies the conditions:

$$
\begin{gathered}
\left.\frac{\partial u(x, t)}{\partial v_{A}}\right|_{S}=0, \\
u(x, 0)=u_{0}(x), \quad x \in \Omega, \\
u_{t}(x, 0)=u_{1}(x), \quad x \in \Omega, \\
\int_{\Omega} R(x) u(x, T) d x=R_{0}
\end{gathered}
$$

(here $\frac{\partial u(x, t)}{\partial v_{A}}=\alpha^{i j}(x) u_{x_{j}}(x, t) v_{j}(x), v_{j}(x)$ are the components of the inward normal vector to $\Gamma$ at the current point). 
Inverse Problem II: Find a function $u(x, t)$ and a number $b$ such that they satisfy Equation (1) in the cylinder $Q$ (for a given number $a$ ) and the function $u(x, t)$ enjoys conditions (2)-(5).

In Inverse Problems I and II, conditions (2)-(4) are the conditions of the usual second initial boundary value problem for second-order hyperbolic equations, whereas condition (5) is a linear final-integral condition, whose necessity is dictated by the presence in (1) of an additional unknown value-the number $a$ or the number $b$.

If, in Inverse Problems I and II, instead of condition (2), we define conditions of the first or third initial boundary value problems then the essence of the results presented below on the existence and uniqueness of solutions does not change in principle but, in some cases, the cumbersomeness of the calculations increases. We will speak of that in Section 4.

\section{Solvability of Inverse Problems I and II}

Auxiliary constructions are then carried out (first, for Inverse Problem I).

Put:

$$
\begin{gathered}
f_{1}(x, t)=\int_{0}^{t} f(x, \tau) d \tau+u_{1}(x), \\
R_{1}=\int_{\Omega} R(x) u_{0}(x) d x, \quad F_{1}=\int_{\Omega} R(x) f_{1}(x, T) d x .
\end{gathered}
$$

Further, denote by $A_{0}$ the operator $A-\alpha^{0}(x)$.

Equation (1) can be written down in the integro-differential form:

$$
u_{t}(x, t)-\int_{0}^{t} A_{0} u(x, \tau) d \tau+a\left[u(x, t)-u_{0}(x)\right]+\int_{0}^{t}\left[b-\alpha^{0}(x)\right] u(x, \tau) d \tau=f_{1}(x, t) .
$$

Put in $\left(1^{\prime}\right) t=T$, multiply by $R(x)$, and integrate over $\Omega$. We then obtain the equality:

$$
\begin{gathered}
\int_{\Omega} R(x) u_{t}(x, T) d x-\int_{Q} R(x) A_{0} u(x, t) d x d t+a\left(R_{0}-R_{1}\right) \\
+\int_{Q}\left[b-\alpha^{0}(x)\right] R(x) u(x, t) d x d t=F_{1} .
\end{gathered}
$$

Given a function $w(x, t)$, we put:

$$
\begin{gathered}
\varphi_{1}(w)=\int_{Q} R(x) A w(x, t) d x d t-b \int_{Q} R(x) w(x, t) d x d t \\
-\int_{\Omega} R(x) w_{t}(x, T) d x .
\end{gathered}
$$

Suppose the fulfillment of the condition:

$$
R_{0}>0, \quad R_{1}<R_{0}
$$

This condition enables us to obtain a representation of the number $a$ :

$$
a=\frac{1}{R_{0}-R_{1}}\left[F_{1}+\varphi_{1}(u)\right]
$$


Consider an auxiliary boundary value problem: Find a function $u(x, t)$ that is a solution in the cylinder $Q$ to the equation:

$$
u_{t t}-A u+\frac{F_{1}+\varphi_{1}(u)}{R_{0}-R_{1}} u_{t}+b u=f(x, t)
$$

and satisfies conditions (2)-(4). This problem is a second initial boundary value problem for a nonlinear "loaded" differential equation (see $[33,34])$. The solvability of boundary value problems for such equations has not been studied before.

It is a solution $u(x, t)$ to the auxiliary problem (8), (2)-(4) that will enable us to construct a solution to Inverse Problem I.

Introduce the notations:

$$
\begin{gathered}
f_{2}(x, t)=(T-t) f(x, t) \\
N_{1}=2\left\|f_{2}\right\|_{L_{2}(Q)} \\
N_{2}=\int_{\Omega}\left\{u_{1}^{2}(x)+\alpha^{i j}(x) u_{0 x_{i}}(x) u_{0 x_{j}}(x)+\left[b-\alpha^{0}(x)\right] u_{0}^{2}(x)\right\} d x, \\
N_{3}=T N_{2}, \quad N_{4}=\frac{1}{2}\left(N_{1}+\sqrt{N_{1}^{2}+4 N_{3}}\right), \\
N_{5}=2\left(\frac{N_{4}^{2}}{T}+N_{4}\|f\|_{L_{2}(Q)}\right), \\
N_{6}=T^{1 / 2} N_{4}\left\{\left(\int_{\Omega} \alpha^{i j}(x) R_{x_{i}}(x) R_{x_{j}}(x) d x\right)^{1 / 2}\right. \\
\left.+\left(\int_{\Omega}\left[b-\alpha^{0}(x)\right] R^{2}(x) d x\right)^{1 / 2}\right\}+N_{5}^{1 / 2}\|R\|_{L_{2}(\Omega)} .
\end{gathered}
$$

Theorem 1. Let the functions $f(x, t), u_{0}(x)$, and $u_{1}(x)$ be such that $f(x, t) \in L_{2}(Q), f_{t}(x, t) \in L_{2}(Q)$, $u_{0}(x) \in W_{2}^{2}(\Omega), u_{1}(x) \in W_{2}^{1}(\Omega)$ and suppose the fulfillment of condition (6) and also of the condition:

$$
\begin{gathered}
\alpha^{i j}(x) \in C^{1}(\bar{\Omega}), \quad \alpha^{0}(x) \in C(\bar{\Omega}), \quad R(x) \in C^{1}(\bar{\Omega}) ; \\
\alpha^{i j}(x)=\alpha^{j i}(x), \quad i, j=1, \ldots, n, \quad x \in \bar{\Omega} ; \\
\alpha^{i j}(x) \xi_{i} \xi_{j} \geq k_{0}|\xi|^{2}, \quad k_{0}>0, \quad x \in \bar{\Omega}, \quad \xi \in \mathbb{R}^{n} ; \\
\alpha^{0}(x) \leq 0 \quad \text { for } \quad x \in \bar{\Omega} ; \\
\frac{\partial u_{0}(x)}{\partial v_{A}}=\frac{\partial u_{1}(x)}{\partial v_{A}}=0 \quad \text { for } \quad x \in \Omega ; \\
F_{1}>0, \quad b \geq 0 ; \\
N_{6}<F_{1} .
\end{gathered}
$$

Then the boundary value problem (8), (2)-(4) has a solution $u(x, t)$ such that $u(x, t) \in L_{\infty}\left(0, T ; W_{2}^{2}(\Omega)\right)$, $u_{t}(x, t) \in L_{\infty}\left(0, T ; W_{2}^{1}(\Omega)\right), u_{t t}(x, t) \in L_{\infty}\left(0, T ; L_{2}(\Omega)\right)$.

Proof. Make use of the truncation method, the fixed point method, and the regularization method.

For a number $\beta \in\left(0, F_{1}\right]$ (the exact value of $\beta$ will be specified below), define the truncating function $G_{\beta}(\xi)$ : 


$$
G_{\beta}(\xi)=\left\{\begin{array}{rll}
\xi & \text { if } & |\xi|<\beta \\
\beta & \text { if } & \xi \geq \beta, \\
-\beta & \text { if } & \xi \leq-\beta .
\end{array}\right.
$$

Consider the boundary value problem: Find a function $u(x, t)$ that is a solution in the cylinder $Q$ to the equation:

$$
u_{t t}-A u+\frac{F_{1}+G_{\beta}\left(\varphi_{1}(u)\right)}{R_{0}-R_{1}} u_{t}+b u=f(x, t)
$$

and satisfies conditions (2)-(4). Establish the solvability of this problem with the use of the regularization method and the fixed point method.

Let $\varepsilon$ be a positive number. Consider the boundary value problem: Find a function $u(x, t)$ that is a solution in $Q$ to the equation:

$$
u_{t t}-A u+\frac{F_{1}+G_{\beta}\left(\varphi_{1}(u)\right)}{R_{0}-R_{1}} u_{t}+b u-\varepsilon A u_{t}=f(x, t)
$$

and satisfies conditions (2)-(4). Using the fixed point method, demonstrate that, for fixed $\varepsilon$ and $f(x, t)$ belonging to $L_{2}(Q)$, this problem has a regular solution (i.e., a solution having all weak derivatives in the sense of Sobolev occurring in the equation).

Denote by $V$ the linear space:

$$
\begin{gathered}
V=\left\{v(x, t): v(x, t) \in L_{\infty}\left(0, T ; W_{2}^{2}(\Omega)\right),\right. \\
\left.v_{t}(x, t) \in L_{\infty}\left(0, T ; W_{2}^{2}(\Omega)\right), v_{t t}(x, t) \in L_{2}(Q)\right\} .
\end{gathered}
$$

Endow this space with the norm:

$$
\|v\|_{V}=\left(\|v\|_{L_{\infty}\left(0, T ; W_{2}^{2}(\Omega)\right)}^{2}+\left\|v_{t}\right\|_{L_{\infty}\left(0, T ; W_{2}^{2}(\Omega)\right)}^{2}+\left\|v_{t t}\right\|_{L_{2}(Q)}^{2}\right)^{1 / 2} .
$$

Obviously, with this norm, $V$ is a Banach space.

Let $w(x, t)$ be a function in $V$. Consider the problem: Find a function $u(x, t)$ that is a solution in $Q$ to the equation:

$$
u_{t t}-A u+\frac{F_{1}+G_{\beta}\left(\varphi_{1}(w)\right)}{R_{0}-R_{1}} u_{t}+b u-\varepsilon A u_{t}=f
$$

and satisfies conditions (2)-(4). As is well known (see [3,35,36]), for fixed $\varepsilon$ and $f(x, t) \in L_{2}(Q)$, this boundary value problem has a solution $u(x, t)$ belonging to $V$ (this fact is not hard to also prove directly with the use of the classical Galerkin method with the choice of a special basis [37]). Consequently, it generates an operator $\Phi$ taking $V$ into itself: $\Phi(w)=u$. Show that the operator $\Phi$ has fixed points in $V$.

Observe first of all that, under the hypotheses of the theorem and for $f(x, t) \in L_{2}(Q)$, all possible solutions $u(x, t)$ to the boundary value problem $\left(8_{\varepsilon, w}^{\prime}\right),(2)-(4)$ satisfy the estimate:

$$
\|v\|_{V} \leq C_{0}
$$

with a constant $C_{0}$ defined only by the functions $f(x, t), R(x), u_{0}(x), u_{1}(x), \alpha^{i j}(x), i, j=1, \ldots, n$, $\alpha^{0}(x)$, the domain $\Omega$, and also the numbers $b, \beta, T, R_{0}$, and $\varepsilon$. This estimate in particular implies that the operator $\Phi$ takes any ball of a radius greater than $C_{0}$ into itself.

Show that $\Phi$ is continuous on each such ball.

Let $\left\{w_{m}(x, t)\right\}_{m=1}^{\infty}$ be a sequence of functions in $V$ converging in $V$ to a function $\bar{w}_{0}(x, t), u_{m}(x, t)$, $\bar{u}_{0}(x, t)$ are the images of $w_{m}(x, t)$ and $\bar{w}_{0}(x, t)$ under the action of $\Phi$. We have the equalities: 


$$
\begin{gathered}
u_{m t t}-\bar{u}_{0 t t}-A\left(u_{m}-\bar{u}_{0}\right)+\frac{F_{1}+G_{\beta}\left(\varphi_{1}\left(\bar{u}_{0}\right)\right)}{R_{0}-R_{1}}\left(u_{m t}-\bar{u}_{0 t}\right)+b\left(u_{m}-\bar{u}_{0}\right) \\
=\frac{G_{\beta}\left(\varphi_{1}\left(\bar{u}_{0}\right)\right)-G_{\beta}\left(\varphi_{1}\left(u_{m}\right)\right)}{R_{0}-R_{1}} u_{m t}, \quad(x, t) \in Q, \\
\frac{\partial u_{m}(x, t)}{\partial v_{A}}-\left.\frac{\partial \bar{u}_{0}(x, t)}{\partial v_{A}}\right|_{S}=0, \\
u_{m}(x, 0)-\bar{u}_{0}(x, 0)=0, \quad u_{m t}(x, 0)-\bar{u}_{0 t}(x, 0)=0 \quad \text { for } \quad x \in \Omega .
\end{gathered}
$$

These equalities mean that the functions $u_{m}(x, t)-\bar{u}_{0}(x, t)$ are a solution to the second initial boundary value problem for the pseudohyperbolic Equation (17). Repeating for this problem the proof of (16) and taking into account that the function $G_{\beta}(\xi)$ is Lipschitz continuous and that the function family $\left\{u_{m}(x, t)\right\}_{m=1}^{\infty}$ is uniformly bounded in $V$, we obtain the inequality:

$$
\left\|u_{m}-\bar{u}_{0}\right\|_{V} \leq C_{1}\left|\varphi_{1}\left(w_{m}-w_{0}\right)\right|
$$

with a number $C_{1}$ defined only by the functions $f(x, t), R(x), \alpha^{i j}(x), i, j=1, \ldots, n, \alpha^{0}(x)$, the domain $\Omega$, and also by the numbers $b, \beta, T, R_{0}$, and $\varepsilon$.

We have the equalities:

$$
\begin{gathered}
\varphi_{1}\left(w_{m}-w_{0}\right)=-\int_{Q} \alpha^{i j}(x) R_{x_{i}}(x)\left(w_{m}-w_{0}\right)_{x_{j} t} d x d t \\
-\int_{S} R(x) \alpha^{i j}(x)\left(w_{m}-w_{0}\right)_{x_{j}} v_{i} d s d t+\int_{Q}\left(\alpha^{0}(x)-b\right) R(x)\left(w_{m}-w_{0}\right) d x d t \\
-\int_{\Omega} R(x)\left[w_{m t}(x, T)-w_{0 t}(x, T)\right] d x .
\end{gathered}
$$

These equalities and the convergence in $V$ of the sequence $\left\{w_{m}(x, t)\right\}_{m=1}^{\infty}$ to $w(x, t)$ imply that the right-hand side of (20) tends to zero as $m \rightarrow \infty$. This means that $\Phi$ is continuous on the ball of radius $C_{0}$ of the space $V$.

Let us now prove that the operator $\Phi$ is compact on the ball of radius $C_{0}$ in $V$.

Let $\left\{w_{m}(x, t)\right\}_{m=1}^{\infty}$ be a family of functions in the above-mentioned ball and let $\left\{u_{m}(x, t)\right\}_{m=1}^{\infty}$ be the corresponding family of the images of the functions $w_{m}(x, t)$ under $\Phi$. The boundedness of the family $\left\{w_{m}(x, t)\right\}_{m=1}^{\infty}$ in $V$ means in particular that the families $\left\{w_{m x_{i}}(x, t)\right\}_{m=1}^{\infty}, i=1, \ldots, n$, $\left\{w_{m t}(x, t)\right\}_{m=1}^{\infty}$ are uniformly bounded in $W_{2}^{1}(Q)$ and the family $\left\{w_{m t}(x, T)\right\}_{m=1}^{\infty}$ is uniformly bounded in $W_{2}^{1}(\Omega)$. The classical embedding theorems (see $[31,32,38]$ ) imply that there exists a sequence $\left\{w_{m_{k}}(x, t)\right\}_{k=1}^{\infty}$ such that the sequences $\left\{w_{m_{k} x_{i}}(x, t)\right\}_{k=1}^{\infty}, i=1, \ldots, n$, converge strongly in $L_{2}(Q)$ and $L_{2}(S)$, and the sequence $\left\{w_{m_{k} t}(x, T)\right\}_{k=1}^{\infty}$ converges strongly in $L_{2}(\Omega)$. The functions $w_{m_{k}}(x, t)-$ $w_{m_{l}}(x, t)$ and $u_{m_{k}}(x, t)-u_{m_{l}}(x, t)(k, l$ are naturals) satisfy the equalities:

$$
\begin{gathered}
u_{m_{k} t t}-u_{m_{l} t t}-A\left(u_{m_{k}}-u_{m_{l}}\right)+\frac{F_{1}+G_{\beta}\left(\varphi_{1}\left(w_{m_{k}}\right)\right)}{R_{0}-R_{1}}\left(u_{m_{k} t}-u_{m_{l} t}\right)+b\left(u_{m_{k}}-u_{m_{l}}\right) \\
=\frac{G_{\beta}\left(\varphi_{1}\left(w_{m_{l}}\right)\right)-G_{\beta}\left(\varphi_{1}\left(w_{m_{k}}\right)\right)}{R_{0}-R_{1}} u_{m_{l} t} \\
\frac{\partial u_{m_{k}}(x, t)}{\partial v_{A}}-\left.\frac{\partial u_{m_{l}}(x, t)}{\partial v_{A}}\right|_{S}=0, \\
u_{m_{k}}(x, 0)-u_{m_{l}}(x, 0)=0, \quad u_{m_{k} t}(x, 0)-u_{m_{l} t}(x, 0)=0 \text { for } x \in \Omega .
\end{gathered}
$$


Repeating the proof of (16) and reckoning with the fact that the sequences $\left\{w_{m_{k}} x_{i}(x, t)\right\}_{k=1}^{\infty}$, $i=1, \ldots, n$, are Cauchy sequences in $L_{2}(Q)$ and $L_{2}(S)$, the sequence $\left\{u_{m_{k} t}(x, T)\right\}_{k=1}^{\infty}$ is a Cauchy sequence in $L_{2}(\Omega)$, we conclude that these equalities imply that $\left\{u_{m_{k}}(x, t)\right\}_{k=1}^{\infty}$ is a Cauchy sequence in $V$. In other words, what has been proved implies that, for any sequence $\left\{w_{m}(x, t)\right\}_{m=1}^{\infty}$ from the ball of radius $C_{0}$ in $V$, from the sequence $\left\{\Phi\left(w_{m}\right)\right\}_{m=1}^{\infty}$, one can extract a strongly convergent sequence in $V$. This means that the operator $\Phi$ is compact on the ball of radius $C_{0}$ in $V$.

Thus, the operator $\Phi$ takes the ball of radius $C_{0}$ in $V$ into itself, and is continuous and compact on this ball. By Schauder's theorem, $\Phi$ has at least one fixed point in the ball of radius $C_{0}: \Phi(u)=u$. This fixed point is a solution from $V$ to the boundary value problem $\left(8_{\varepsilon}^{\prime}\right),(2)-(4)$. Showing that these fixed points satisfy a priori estimates uniform over $\varepsilon$.

Multiply Equation $\left(8_{\varepsilon}^{\prime}\right)$ by the function $(T-t) u_{t}$ and integrate over the cylinder $Q$. Using the boundary conditions and the hypotheses of the theorem and applying Hölder's inequality, we get the inequality:

$$
\begin{gathered}
\int_{Q}\left[u_{t}^{2}+\alpha^{i j} u_{x_{i}} u_{x_{j}}+\frac{2\left[F_{1}+G_{\beta}\left(\varphi_{1}(u)\right)\right](T-t)}{R_{0}-R_{1}} u_{t}^{2}+\left(b-\alpha^{0}\right) u^{2}\right] d x d t \\
+2 \varepsilon \int_{Q}(T-t)\left[\alpha^{i j} u_{x_{i}} u_{x_{j} t}-\alpha^{0} u_{t}^{2}\right] d x d t \\
\leq N_{1}\left(\int _ { Q } \left[u_{t}^{2}+\alpha^{i j} u_{x_{i}} u_{x_{j}}+\frac{2\left[F_{1}+G_{\beta}\left(\varphi_{1}(u)\right)\right](T-t)}{R_{0}-R_{1}} u_{t}^{2}\right.\right. \\
\left.\left.+\left(b-\alpha^{0}\right) u^{2}\right] d x d t+2 \varepsilon \int_{Q}(T-t)\left[\alpha^{i j} u_{x_{i} t} u_{x_{j} t}-\alpha^{0} u_{t}^{2}\right] d x d t\right)^{1 / 2}+N_{3} .
\end{gathered}
$$

This inequality implies an a priori estimate of solutions $u(x, t)$ to the boundary value problem $\left(8_{\varepsilon}^{\prime}\right),(2)-(4)$ :

$$
\begin{gathered}
\int_{Q}\left[u_{t}^{2}+\alpha^{i j} u_{x_{i}} u_{x_{j}}+\frac{2\left[F_{1}+G_{\beta}\left(\varphi_{1}(u)\right)\right](T-t)}{R_{0}-R_{1}} u_{t}^{2}+\left(b-\alpha^{0}\right) u^{2}\right] d x d t \\
+2 \varepsilon \int_{Q}(T-t)\left[\alpha^{i j} u_{x_{i} t} u_{x_{j} t}-\alpha^{0} u_{t}^{2}\right] d x d t \leq N_{4}^{2} .
\end{gathered}
$$

At the next step, multiply $\left(8_{\varepsilon}^{\prime}\right)$ by the function $-(T-2 t) u_{t}(x, t)$ and integrate it over the cylinder $Q$. We obtain the equality:

$$
\begin{gathered}
T \int_{\Omega}\left[u_{t}^{2}(x, T)+\alpha^{i j}(x) u_{x_{i}}(x, T) u_{x_{j}}(x, T)+\left(b-\alpha^{0}\right) u^{2}(x, T)\right. \\
\left.+u_{1}^{2}(x)+\alpha^{i j}(x) u_{0 x_{i}}(x) u_{0 x_{j}}(x)+\left(b-\alpha^{0}(x)\right) u_{0}^{2}(x)\right] d x \\
+\frac{2\left[F_{1}+G_{\beta}\left(\varphi_{1}(u)\right)\right]}{R_{0}-R_{1}} \int_{Q} t u_{t}^{2} d x d t+2 \varepsilon \int_{Q}\left[t\left(\alpha^{i j} u_{x_{i}} u_{x_{j} t}-\alpha^{0} u_{t}^{2}\right)\right] d x d t
\end{gathered}
$$




$$
\begin{gathered}
=2 \int_{Q}\left[u_{t}^{2}+\alpha^{i j} u_{x_{i}} u_{x_{j}}+\frac{F_{1}+G_{\beta}\left(\varphi_{1}(u)\right)}{R_{0}-R_{1}}(T-t) u_{t}^{2}+\left(b-\alpha^{0}\right) u^{2}\right] d x d t \\
+2 \varepsilon \int_{Q}(T-t)\left[\alpha^{i j} u_{x_{i} t} u_{x_{j} t}-\alpha^{0} u_{t}^{2}\right] d x d t .
\end{gathered}
$$

This equality and estimate (21) imply the inequality:

$$
\int_{\Omega} u_{t}^{2}(x, T) d x \leq N_{5}
$$

Estimates (21) and (22) imply the boundedness of $\left|\varphi_{1}(u)\right|$ :

$$
\left|\varphi_{1}(u)\right| \leq N_{6}
$$

Fix a number $\beta: \beta=N_{6}$. For such a choice of $\beta$, we have the equality $G_{\beta}\left(\varphi_{1}(u)\right)=\varphi_{1}(u)$. Consequently, a solution $u(x, t)$ to Equation $\left(8_{\varepsilon}^{\prime}\right)$ is a solution to the equation:

$$
u_{t t}-A u+\frac{F_{1}+\varphi_{1}(u)}{R_{0}-R_{1}} u_{t}+b u-\varepsilon A u_{t}=f(x, t) .
$$

Multiply $\left(8_{\varepsilon}^{\prime}\right)$ by the function $(T-t) A u_{t}(x, t)$ and integrate it over $Q$. Using the boundary conditions and inequalities (21) and (23), we conclude that solutions $u(x, t)$ to the boundary value problem $\left(8_{\varepsilon}^{\prime}\right),(2)-(4)$ satisfy the estimate:

$$
\int_{Q}(A u)^{2} d x d t+\varepsilon \int_{Q}\left(A u_{t}\right)^{2} d x d t \leq C_{2}
$$

with a constant $C_{2}$ determined only by the functions $f(x, t), \alpha^{i j}(x), i, j=1, \ldots, n, \alpha^{0}(x), R(x), u_{0}(x)$, and $u_{1}(x)$, and also by the domain $\Omega$ and the numbers $b$ and $T$.

The last estimate:

$$
\int_{Q} u_{t t}^{2} d x d t \leq C_{3}
$$

obviously follows from (21), (23), and (25). The constant $C_{3}$ in this estimate is determined only by the functions $f(x, t), \alpha^{i j}(x), i, j=1, \ldots, n, \alpha^{0}(x), R(x), u_{0}(x)$, and $u_{1}(x)$ and also by the domain $\Omega$ and the numbers $b$ and $T$.

Estimates (21), (25), and (26), the second main inequality for elliptic operators [38], the classical embedding theorems (see the proof of the compactness of $\Phi$ ), and the reflexivity of a Hilbert space imply that there exist sequences $\left\{\varepsilon_{m}\right\}_{m=1}^{\infty}$ of positive numbers, of functions $\left\{u_{m}(x, t)\right\}_{m=1}^{\infty}$ that are solutions to the boundary value problem (24), (2)-(4), and a function $u(x, t)$ such that, as $m \rightarrow \infty$, we have the convergences:

$$
\begin{aligned}
\varepsilon_{m} & \rightarrow 0, \\
\varphi_{1}\left(u_{m}\right) & \rightarrow \varphi_{1}(u), \\
u_{m}(x, t) \rightarrow u(x, t) & \text { weakly in } W_{2}^{2}(Q), \\
\varepsilon_{m} A u_{m t}(x, t) \rightarrow 0 & \text { weakly in } L_{2}(Q) .
\end{aligned}
$$

Obviously, the limit function $u(x, t)$ is a solution to the boundary value problem (8), (2)-(4). Moreover, this solution satisfies the inclusions $u(x, t) \in L_{\infty}\left(0, T ; W_{2}^{2}(\Omega)\right), u_{t}(x, t) \in L_{\infty}\left(0, T ; W_{2}^{1}(\Omega)\right)$, $u_{t t}(x, t) \in L_{\infty}\left(0, T ; L_{2}(\Omega)\right)$.

The theorem is proved. 
Theorem 2. Let the functions $f(x, t), u_{0}(x)$, and $u_{1}(x)$ be such that $f(x, t) \in L_{2}(Q), f_{t}(x, t) \in L_{2}(Q)$, $\left.u_{0}(x) \in W_{2}^{2}(\Omega)\right), u_{1}(x) \in W_{2}^{1}(\Omega)$, and suppose the fulfillment of conditions (6), (9)-(15). Then Inverse Problem I has solution $\{u(x, t), a\}$ such that:

$$
\begin{gathered}
u(x, t) \in L_{\infty}\left(0, T ; W_{2}^{2}(\Omega)\right), \quad u_{t}(x, t) \in L_{\infty}\left(0, T ; W_{2}^{1}(\Omega)\right), \\
u_{t t}(x, t) \in L_{\infty}\left(0, T ; L_{2}(\Omega)\right), \quad a>0 .
\end{gathered}
$$

Proof. Let $u(x, t)$ be a solution to the boundary value problem (8), (2)-(4). Define the number $a$ by (7). Obviously, this number and the function $u(x, t)$ are related by Equation (1) in $Q$. Show that $u(x, t)$ satisfies the overdetermination condition (5).

Write down Equation (1) in integro-differential form (in the form of Equation (1')), put $t=T$ therein, multiply it by $R(x)$, and integrate over $\Omega$. We obtain the equality:

$$
\frac{F_{1}+\varphi_{1}(u)}{R_{0}-R_{1}}\left[\int_{\Omega} R(x) u(x, T) d x-R_{0}\right]=F_{1}+\varphi_{1}(u) .
$$

Since $F_{1}+\varphi_{1}(u) \neq 0$, we obtain the required condition:

$$
\int_{\Omega} R(x) u(x, T) d x=R_{0} .
$$

All what was said above means that the function $u(x, t)$, which is a solution to the boundary value problem (8), (2)-(4), and the number $a$ defined from $u(x, t)$ by (7) give a desired solution $\{u(x, t), a\}$ to Inverse Problem I.

The theorem is proved.

Turn to investigating Inverse Problem II without separating a theorem on the solvability of the initial boundary value problem for the corresponding loaded equation.

The study of Inverse Problem II will again be carried out with the use of passing to a special "loaded" differential equation, investigating the solvability of the corresponding initial boundary value problem, and then constructing a solution to the initial inverse problem. The solvability of the auxiliary boundary value problem will again be established with the use of the regularization method, the fixed point method, and a priori estimates.

Suppose the fulfillment of the condition:

$$
R_{0}>0
$$

From a fixed function $w(x, t)$, define a function $\varphi_{2}(w)$ :

$$
\varphi_{2}(w)=\int_{\Omega} R(x)\left[w_{t t}(x, T)-A w(x, T)+a w_{t}(x, T)\right] d x
$$

Then define a number $F_{2}$ :

$$
F_{2}=\int_{\Omega} R(x) f(x, T) d x
$$

Put $t=T$ in Equation (1). Condition (27) makes it possible to obtain a representation of the number $b$ :

$$
b=\frac{F_{2}-\varphi_{2}(u)}{R_{0}}
$$

Consider the "loaded" differential equation: 


$$
u_{t t}-A u+a u_{t}+\frac{F_{2}-\varphi_{2}(u)}{R_{0}} u=f(x, t) .
$$

A solution $u(x, t)$ to the second boundary value problem for this equation will enable us to construct a solution of Inverse Problem II.

Put:

$$
\begin{aligned}
& M_{1}=\frac{1}{a} \int_{Q} f^{2} d x d t \\
& +\int_{\Omega}\left[u_{1}^{2}(x)+\alpha^{i j}(x) u_{0 x_{i}}(x) u_{0 x_{j}}(x)+\left(\frac{F_{2}}{R_{0}}-\alpha^{0}(x)\right) u_{0}^{2}(x)\right] d x, \\
& M_{2}=\frac{1}{R_{0}} \int_{\Omega} u_{0}^{2}(x) d x\left[a\|R\|_{L_{2}(\Omega)}+\left(\int_{\Omega} \alpha^{i j}(x) R_{x_{i}}(x) R_{x_{j}}(x) d x\right)^{1 / 2}\right. \\
& \left.+\left(\int_{\Omega}\left|\alpha^{0}(x)\right| R^{2}(x) d x\right)^{1 / 2}\right] \\
& M_{3}=\frac{1}{R_{0}}\left\|u_{0}\right\|_{L_{2}(\Omega)}^{2}\|R\|_{L_{2}(\Omega)} \\
& M_{4}=2 M_{1}+M_{2}^{2} \\
& v_{0}(x)=f(x, 0)+A u_{0}(x)-a u_{1}(x)-\frac{F_{2}}{R_{0}} u_{0}(x), \\
& M_{5}=\frac{1}{a} \int_{Q} f_{t}^{2} d x d t+\int_{\Omega}\left[\alpha^{i j}(x) u_{1 x_{i}}(x) u_{1 x_{j}}(x)+\left(\frac{F_{2}}{R_{0}}-\alpha^{0}(x)\right) u_{1}^{2}(x)\right] d x \\
& +2\left\|v_{0}\right\|_{L_{2}(\Omega)}^{2}+\frac{1}{2 R_{0}^{2}}\left\|u_{1}\right\|_{L_{2}(\Omega)}^{4}, \\
& M_{6}=\frac{1}{R_{0}^{2}}\left(2\left\|u_{0}\right\|_{L_{2}(\Omega)}^{2}+\frac{1}{2}\left\|u_{1}\right\|_{L_{2}(\Omega)}^{4}\right) \\
& M_{7}=2 M_{6}\|R\|_{L_{2}(\Omega)}^{2} \\
& M_{8}=2 M_{6}\left[a\|R\|_{L_{2}(\Omega)}+\left(\int_{\Omega} \alpha^{i j}(x) R_{x_{i}}(x) R_{x_{j}}(x) d x\right)^{1 / 2}\right. \\
& \left.+\left(\int_{\Omega}\left|\alpha^{0}(x)\right| R^{2}(x) d x\right)^{1 / 2}\right] \\
& M_{9}=\frac{M_{5}}{1-M_{7}}, \quad M_{10}=\frac{M_{8}}{1-M_{7}}, \\
& M_{11}=M_{9}+M_{4} M_{10}, \quad M_{12}=2 M_{3} M_{10}, \\
& M_{13}=\frac{1}{2}\left(M_{12}+\sqrt{M_{12}^{2}+4 M_{11}}\right), \\
& M_{14}=M_{4}+2 M_{3} M_{13}^{1 / 2} .
\end{aligned}
$$




$$
\begin{gathered}
M_{15}=M_{13}^{1 / 2}\|R\|_{L_{2}(\Omega)}+\left[a\|R\|_{L_{2}(\Omega)}+\left(\int_{\Omega} \alpha^{i j}(x) R_{x_{i}}(x) R_{x_{j}}(x) d x\right)^{1 / 2}\right. \\
\left.+\left(\int_{\Omega}\left|\alpha^{0}(x)\right| R^{2}(x) d x\right)^{1 / 2}\right] M_{14}^{1 / 2}
\end{gathered}
$$

Theorem 3. Let the functions $f(x, t), u_{0}(x)$, and $u_{1}(x)$ be such that $f(x, t) \in L_{2}\left(0, T ; W_{2}^{1}(\Omega)\right)$, $f_{t}(x, t) \in L_{2}(Q), u_{0}(x) \in W_{2}^{2}(\Omega), u_{1}(x) \in W_{2}^{1}(\Omega)$. Moreover, suppose the fulfillment of conditions (9)-(13), (27), and also the conditions:

$$
\begin{gathered}
F_{2}>0, \quad b \geq 0 ; \\
M_{7}<1, \quad M_{15}<F_{2} .
\end{gathered}
$$

Then Inverse Problem II has a solution $\{u(x, t), b\}$ such that:

$$
\begin{gathered}
u(x, t) \in L_{\infty}\left(0, T ; W_{2}^{2}(\Omega)\right), \quad u_{t}(x, t) \in L_{\infty}\left(0, T ; W_{2}^{1}(\Omega)\right), \\
u_{t t}(x, t) \in L_{\infty}\left(0, T ; L_{2}(\Omega)\right), \quad b>0 .
\end{gathered}
$$

Proof. Let $\beta$ be a number in $\left(0, F_{2}\right]$ and $G_{\beta}(\xi)$ be the truncating function defined in proving Theorem 1 . Using the regularized equation:

$$
u_{t t}-A u+a u_{t}+\frac{F_{2}-G_{\beta}\left(\varphi_{2}(u)\right)}{R_{0}} u-\varepsilon A u_{t}=f(x, t),
$$

the fixed point method, a priori estimates of solutions to initial boundary value problems for pseudohyperbolic equations and also using the standard procedure of choosing a convergent sequence (based on the reflexivity of a Hilbert space), it is not hard to prove that, under conditions (9)-(13) and (27), the initial boundary value problem for the equation:

$$
u_{t t}-A u+a u_{t}+\frac{F_{2}-G_{\beta}\left(\varphi_{2}(u)\right)}{R_{0}} u=f(x, t)
$$

with conditions (2)-(4) has a solution $u(x, t)$ such that $u(x, t) \in L_{\infty}\left(0, T ; W_{2}^{2}(\Omega)\right), u_{t}(x, t) \in$ $L_{\infty}\left(0, T ; W_{2}^{1}(\Omega)\right), u_{t t}(x, t) \in L_{\infty}\left(0, T ; L_{2}(\Omega)\right)$. Show that the solutions to this problem admit a priori estimates sufficient for constructing a solution to Inverse Problem II.

For convenience, introduce the notations:

$$
\begin{gathered}
I_{1}=\int_{\Omega} u_{t t}^{2}(x, T) d x \\
I_{2}=\int_{\Omega}\left[u_{t}^{2}(x, T)+\alpha^{i j}(x) u_{x_{i}}(x, T) u_{x_{j}}(x, T)-\alpha^{0}(x) u^{2}(x, T)\right] d x .
\end{gathered}
$$

Multiply Equation $\left(1^{\prime \prime}\right)$ by the function $u_{t}(x, t)$ and integrate over the cylinder $Q$. Using the boundary conditions and applying Hölder's inequality, it is not hard to conclude that a solution $u(x, t)$ to the boundary value problem $\left(1^{\prime \prime}\right),(2)-(4)$ satisfies the inequality:

$$
I_{2}+a \int_{Q} u_{t}^{2} d x d t \leq \frac{1}{a} \int_{Q} f^{2} d x d t
$$




$$
\begin{gathered}
+\int_{\Omega}\left[u_{1}^{2}(x)+\alpha^{i j}(x) u_{0 x_{i}}(x) u_{0 x_{j}}(x)+\left(\frac{F_{2}}{R_{0}}-\alpha^{0}(x)\right) u_{0}^{2}(x)\right] d x \\
+\frac{\left|\varphi_{2}(u)\right|}{R_{0}}\left\|u_{0}\right\|_{L_{2}(\Omega)}^{2} .
\end{gathered}
$$

We have the inequality:

$$
\begin{gathered}
\left|\varphi_{2}(u)\right| \leq\|R\|_{L_{2}(\Omega)} I_{1}^{1 / 2}+\left[a\|R\|_{L_{2}(\Omega)}+\left(\int_{\Omega} \alpha^{i j}(x) R_{x_{i}}(x) R_{x_{j}}(x) d x\right)^{1 / 2}\right. \\
\left.+\left(\int_{\Omega}\left|\alpha^{0}(x)\right| R^{2}(x) d x\right)^{1 / 2}\right] I_{2}^{1 / 2} .
\end{gathered}
$$

Relations (28) and (29) imply the estimate:

$$
I_{2} \leq M_{4}+2 M_{3} I_{1}^{1 / 2}
$$

The solutions $u(x, t)$ to the boundary value problem $\left(1^{\prime \prime}\right),(2)-(4)$ satisfy the equality:

$$
u_{t t}(x, 0)=v_{0}(x)+\frac{\varphi_{2}(u)}{R_{0}} u_{0}(x) .
$$

This equality implies:

$$
\int_{\Omega} u_{t t}^{2}(x, 0) d x \leq 2\left\|v_{0}\right\|_{L_{2}(\Omega)}^{2}+\frac{2 \varphi_{2}^{2}(u)}{R_{0}^{2}}\left\|u_{0}\right\|_{L_{2}(\Omega)}^{2} .
$$

Furthermore, we have the equality:

$$
\begin{gathered}
\frac{1}{2} \int_{\Omega} u_{t t}^{2}(x, T) d x+\frac{1}{2} \int_{\Omega}\left[\alpha^{i j}(x) u_{x_{i} t}(x, T) u_{x_{j} t}(x, T)-\alpha^{0}(x) u_{t}^{2}(x, T)\right] d x \\
+a \int_{Q} u_{t t}^{2} d x d t+\frac{F_{2}-G_{\beta}\left(\varphi_{2}(u)\right)}{2 R_{0}} \int_{\Omega} u^{2}(x, T) d x \\
=\int_{Q} f_{t} u_{t t} d x d t+\frac{1}{2} \int_{\Omega} u_{t t}^{2}(x, 0) d x \\
+\frac{1}{2} \int_{\Omega}\left[\alpha^{i j}(x) u_{1 x_{i}} u_{1 x_{j}}-\alpha^{0}(x) u_{1}^{2}(x)\right] d x+\frac{F_{2}-G_{\beta}\left(\varphi_{2}(u)\right)}{2 R_{0}} \int_{\Omega} u_{1}^{2}(x) d x
\end{gathered}
$$

(which is easy to validate using passage to the limit in the analogous equality for the regularized equation). Applying Young's inequality and estimate (31), it is not hard to pass from this equality to the inequality:

$$
I_{1} \leq M_{5}+M_{6} \varphi_{2}^{2}(u) .
$$

Using (31) and (29), we infer:

$$
I_{1} \leq M_{5}+M_{6}\left\{2\|R\|_{L_{2}(\Omega)}^{2} I_{1}+2\left[a\|R\|_{L_{2}(\Omega)}+\left(\int_{\Omega} \alpha^{i j}(x) R_{x_{i}}(x) R_{x_{j}}(x) d x\right)^{1 / 2}\right.\right.
$$




$$
\left.\left.+\left(\int_{\Omega}\left|\alpha^{0}(x)\right| R^{2}(x) d x\right)^{1 / 2}\right]^{2} I_{2}\right\}=M_{5}+M_{7} I_{1}+M_{8} I_{2} .
$$

The condition $M_{7}<1$ gives the inequality:

$$
I_{1} \leq M_{9}+M_{10} I_{2} .
$$

From (30) and (32) we obtain:

$$
I_{1} \leq M_{11}+M_{12} I_{1}^{1 / 2},
$$

which implies the estimate:

$$
I_{1} \leq M_{13} .
$$

This estimate and (30) in turn imply that $I_{2}$ is bounded:

$$
I_{2} \leq M_{14}
$$

Finally, estimates (33) and (34) together with (29) give the following inequality for the solutions $u(x, t)$ to the boundary value problem $\left(1^{\prime \prime}\right),(2)-(4)$ :

$$
\left|\varphi_{2}(u)\right| \leq M_{15} .
$$

Fix $\beta: \beta=M_{15}$. For such a choice of the parameter $\beta$, we have the equality $G_{\beta}\left(\varphi_{2}(u)\right)=\varphi_{2}(u)$. Therefore, a solution $u(x, t)$ to the boundary value problem $\left(1^{\prime \prime}\right),(2)-(4)$ is a solution to the equation:

$$
u_{t t}-A u+a u_{t}+\frac{F_{2}-\varphi_{2}(u)}{R_{0}} u=f(x, t) .
$$

Defining the number $b$ by the equality:

$$
b=\frac{F_{2}-\varphi_{2}(u)}{R_{0}},
$$

we obtain a desired solution $\{u(x, t), b\}$ to Inverse Problem II (the fulfillment of the determination condition (5) for $u(x, t)$ is proved as in Theorem 2).

The theorem is proved.

\section{Uniqueness of Solutions}

In Inverse Problem I, consider the case of $\alpha^{0}(x) \equiv 0, b=0$ (the general case differs from this only by more cumbersome calculations and conditions). Let $K_{0}$ be a fixed positive number. Define the set:

$$
\begin{gathered}
V_{K_{0,1}}=\left\{v(x, t): v(x, t) \in L_{\infty}\left(0, T ; W_{2}^{2}(\Omega)\right), \quad v_{t}(x, t) \in L_{\infty}\left(0, T ; W_{2}^{1}(\Omega)\right),\right. \\
\left.v_{t t}(x, t) \in L_{\infty}\left(0, T ; L_{2}(\Omega)\right), \quad\left\|v_{t}(x, t)\right\|_{L_{2}(Q)} \leq K_{0}\right\} .
\end{gathered}
$$

Next, put:

$$
N_{0}=T^{1 / 2}\left(\int_{\Omega} \alpha^{i j}(x) R_{x_{i}}(x) R_{x_{j}}(x) d x\right)^{1 / 2}+\|R\|_{L_{2}(\Omega)} .
$$

Theorem 4. Suppose the fulfillment of all hypotheses of Theorem 1 and also of the conditions:

$$
\alpha^{0}(x) \equiv 0 \quad \text { for } \quad x \in \Omega, \quad b=0 ;
$$




$$
\frac{2 K_{0} N_{0}(T+1)}{R_{0}-R_{1}}<1 .
$$

Then two solutions $\left\{u_{1}(x, t), a_{1}\right\}$ and $\left\{u_{2}(x, t), a_{2}\right\}$ to Inverse Problem I such that $u_{i}(x, t) \in V_{K_{0,1}}$ $a_{i}>0, i=1,2$, coincide.

Proof. Since the functions $u_{1}(x, t)$ and $u_{2}(x, t)$ belong to the set $V_{K_{0,1}}$, the numbers $a_{1}$ and $a_{2}$ satisfy the equalities:

$$
a_{1}=\frac{F_{1}+\varphi_{1}\left(u_{1}\right)}{R_{0}-R_{1}}, \quad a_{2}=\frac{F_{1}+\varphi_{1}\left(u_{2}\right)}{R_{0}-R_{1}} .
$$

The difference $w(x, t)$ of $u_{1}(x, t)$ and $u_{2}(x, t)$ satisfies the equality:

$$
w_{t t}-A w+a_{1} w_{t}=-\frac{\varphi_{1}(w)}{R_{0}-R_{1}} u_{2 t}
$$

in the cylinder $Q$. Moreover, $w(x, t)$ satisfies the conditions:

$$
\begin{gathered}
\left.\frac{\partial w(x, t)}{\partial v_{A}}\right|_{S}=0, \\
w(x, 0)=w_{t}(x, 0)=0, \quad x \in \Omega .
\end{gathered}
$$

We put:

$$
I=\int_{Q}\left(w_{t}^{2}+\alpha^{i j} w_{x_{i}} w_{x_{j}}\right) d x d t+\int_{\Omega} w_{t}^{2}(x, T) d x .
$$

Multiply (35) by $w_{t}(x, t)$ and integrate it over $Q$. Using (36) and (37), applying Hölder's inequality, and reckoning with the membership of the function $u_{2}(x, t)$ in $V_{K_{0,1}}$, we get the inequality

$$
\int_{\Omega} w_{t}^{2}(x, T) d x \leq \frac{2 N_{0} K_{0}}{R_{0}-R_{1}} I .
$$

At the next step, multiply (35) by $(T-t) w_{t}(x, t)$ and integrate it over $Q$. Using (36) and (37) again, applying Hölder's inequality, and reckoning with the membership of $u_{2}(x, t)$ in $V_{K_{0,1}}$, we conclude that $w(x, t)$ satisfies the inequality:

$$
\int_{Q}\left(w_{t}^{2}+\alpha^{i j} w_{x_{i}} w_{x_{j}}\right) d x d t \leq \frac{2 N_{0} K_{0} T}{R_{0}-R_{1}} I .
$$

Summing up (38) and (39), taking into account the hypothesis, we obtain the equality $I=0$. This equality implies that the functions $u_{1}(x, t)$ and $u_{2}(x, t)$ coincide identically in $Q$. As a consequence, the numbers $a_{1}$ and $a_{2}$ coincide.

The theorem is proved.

The uniqueness of solutions to Inverse Problem II will also be established in some simplified case. The general case will differ from this case only by more cumbersome calculations and conditions.

Define the set:

$$
\begin{aligned}
V_{K_{0,2}}= & \left\{v(x, t): v(x, t) \in L_{\infty}\left(0, T ; W_{2}^{2}(\Omega)\right), \quad v_{t}(x, t) \in L_{\infty}\left(0, T ; W_{2}^{1}(\Omega)\right),\right. \\
& \left.v_{t t}(x, t) \in L_{\infty}\left(0, T ; L_{2}(\Omega)\right), \quad\|v\|_{L_{2}(Q)}^{2}+\left\|v_{t}\right\|_{L_{2}(Q)}^{2} \leq K_{0}\right\} .
\end{aligned}
$$

Theorem 5. Suppose the fulfillment of the hypotheses of Theorem 3 and also of the condition:

$$
\alpha^{0}(x) \equiv 0, \quad u_{0}(x) \equiv 0 \text { for } \quad x \in \Omega ;
$$




$$
\frac{2 K_{0}}{a R_{0}^{2}}\left[a\|R\|_{L_{2}(\Omega)}+\left(\int_{\Omega} \alpha^{i j}(x) R_{x_{i}}(x) R_{x_{j}}(x) d x\right)^{1 / 2}\right]^{2}<1 .
$$

Then two solutions $\left\{u_{1}(x, t), b_{1}\right\}$ and $\left\{u_{2}(x, t), b_{2}\right\}$ to Inverse Problem II such that $u_{i}(x, t) \in V_{K_{0,2}}$ $b_{i}>0, i=1,2$, coincide.

Proof. The difference $w(x, t)$ of the functions $u_{1}(x, t)$ and $u_{2}(x, t)$ satisfies the equalities:

$$
\begin{gathered}
w_{t t}-A w+a w_{t}+b_{1} w=-\frac{\varphi_{2}(w)}{R_{0}} u_{2}, \quad(x, t) \in Q, \\
\left.\frac{\partial w(x, t)}{\partial v_{A}}\right|_{S}=0, \\
w(x, 0)=w_{t}(x, 0)=w_{t t}(x, 0)=0, \quad x \in \Omega .
\end{gathered}
$$

These equalities imply the integral relations:

$$
\begin{gathered}
\int_{\Omega}\left[w_{t}^{2}(x, T)+\alpha^{i j}(x) w_{x_{i}}(x, T) w_{x_{j}}(x, T)\right] d x+2 a \int_{Q} w_{t}^{2} d x d t \\
+b_{1} \int_{\Omega} w^{2}(x, T) d x=-\frac{2 \varphi_{2}(w)}{R_{0}} \int_{Q} u_{2} w_{t} d x d t \\
\int_{\Omega}\left[w_{t t}^{2}(x, T)+\alpha^{i j}(x) w_{x_{i} t}(x, T) w_{x_{j} t}(x, T)\right] d x+2 a \int_{Q} w_{t t}^{2} d x d t \\
+b_{1} \int_{\Omega} w_{t}^{2}(x, T) d x=-\frac{2 \varphi_{2}(w)}{R_{0}} \int_{Q} u_{2 t} w_{t t} d x d t .
\end{gathered}
$$

Put,

$$
I=\int_{\Omega}\left[w_{t}^{2}(x, T)+w_{t t}^{2}(x, T)+\alpha^{i j}(x) w_{x_{i}}(x, T) w_{x_{j}}(x, T)\right] d x .
$$

Summing up (40) and (41), applying Young's inequality and taking into account the membership of $u_{2}(x, t)$ in $V_{K_{0,2}}$, we get the inequality:

$$
I \leq \frac{2 K_{0} \varphi_{2}^{2}(w)}{a R_{0}^{2}}
$$

Now, we have the inequality:

$$
\left|\varphi_{2}(w)\right| \leq\left[(a+1)\|R\|_{L_{2}(\Omega)}+\left(\int_{\Omega} \alpha^{i j}(x) R_{x_{i}}(x) R_{x_{j}}(x) d x\right)^{1 / 2}\right] I^{1 / 2} .
$$

Relations (42) and (43) and the hypothesis of the theorem obviously implies the equality $I=0$. But then the function $w(x, t)$ satisfies the equation:

$$
w_{t t}-A w+a w_{t}=0 .
$$

This equation and the boundary conditions imply that the function $w(x, t)$ is identical zero in $Q$. This means that the functions $u_{1}(x, t)$ and $u_{2}(x, t)$, the numbers $b_{1}$ and $b_{2}$ coincide.

The theorem is proved. 


\section{Comments and Appendices}

1. A large number of notations and numerous connections between constants in the hypotheses of Theorems 1-3 make it necessary to check the nonemptiness of the set of the initial data of Inverse Problems I and II for which all conditions of the corresponding theorems are fulfilled.

Suppose that, in Inverse Problem I, we have $\alpha^{0}(x) \equiv 0, u_{0}(x) \equiv 0$ for $x \in \Omega, b=0, R(x) \equiv 1$ for $x \in \Omega$, and let $u_{1}(x)$ be a function positive in $\bar{\Omega}$ and $f(x, t)$ be an arbitrary nonnegative function in $Q$. The constants $N_{1}, N_{3}-N_{6}$ vanish for $T=0$. Consequently, for such data, the conditions $F_{1}>0, N_{6}<F_{1}$ are fulfilled for small $T$.

For Inverse Problem II, we also consider the case of $\alpha^{0}(x) \equiv 0, u_{0}(x) \equiv 0$ for $x \in \Omega, a=0$, $R(x) \equiv 1$ for $x \in \Omega$. Suppose additionally that the function $u_{1}(x)$ is also identical zero in $\Omega$, $f(x, t)$ is a function such that $f(x, 0) \equiv 0$ for $x \in \Omega, f(x, T) \geq f_{0}>0$ for $x \in \Omega$. For such data, the numbers $M_{2}-M_{4}, M_{6}-M_{8}, M_{10}-M_{12}$ are zero, the number $F_{2}$ is positive, and the condition $M_{7}<1$ is fulfilled automatically. The fulfillment of the condition $M_{15}<F_{2}$ is not hard to achieve assuming that the number $f_{0}$ is great.

More complicated examples can be given but those exposed above are quite enough for saying that the set of initial data for which all hypotheses of the corresponding existence theorems are satisfied is not empty.

2. Using the methods presented in this article, it is not hard to examine the solvability of Inverse Problems I and II with the conditions of the first and third initial boundary value problems on the surface $S$. For inverse problems with the condition of the first initial boundary value problem, under the condition:

$$
R(x)=0 \text { for } x \in \Gamma,
$$

all Theorems 1-5 are valid without change. If this condition is not fulfilled then the functions $\varphi_{1}(w)$ and $\varphi_{2}(w)$ have a more complicated form and it is required to obtain some additional a priori estimates (which increases the number of the conditions) but the essence of the result on existence and uniqueness does not change in principle.

If, in Inverse Problems I and II, on the lateral surface $S$, the condition of the third boundary value problem is defined then the technique of proving existence and uniqueness theorems does not change in principle. Only the number of calculations and conditions slightly increases.

3. In Inverse Problems I and II, Equation (1) has a model form. Obviously, this equation can also have a more general form. For instance, the model operator $A$ can be replaced by a general second-order elliptic operator and the numbers $b$ in Inverse Problem I and $a$ in Inverse Problem II can be functions of the variables $x$ and $t$.

Using the above technique, one can study the solvability of Inverse Problems I and II with an operator $A$ that is an elliptic operator of order $2 m$ with all lower terms or a quasielliptic operator (with a natural supplement of the necessary boundary conditions on the surface $S$ ) and the use of the theory of anisotropic spaces [39].

4. Both in the model case considered in the article and in the more general case, the case of Equation (1) with coefficients depending on the variables $x_{1}, \ldots, x_{n}$ and $t$ (which is admitted, as was observed above), the use of the method of separation of variables (the spectral method) faces substantial difficulties.

5. The results on the solvability of initial boundary value problems for nonlinear "loaded" hyperbolic equations obtained in the course of the study have an independent meaning in the author's opinion.

6. Theorems 4 and 5 speak of the uniqueness of a really existing solution. These theorems could be formulated also as independent theorems without binding them to existence theorems.

7. Finally, the last observation: Some constants in the hypothesis of Theorem 3 are defined by the author's choice of the parameters in Young's inequality. Changing these parameters, it is possible to perturb the numbers $M_{i}$. 
Funding: The work is supported by the Mathematical Center in Akademgorodok (Agreement 075-15-2019-1613 with the Ministry of Science and Higher Education of the Russian Federation).

Conflicts of Interest: The author declares no conflict of interest.

\section{References}

1. Prilepko, A.I.; Orlovsky, D.G.; Vasin, I.A. Methods for Solving Inverse Problems in Mathematical Physics; Marcel Dekker: New York, NY, USA, 1999.

2. Kozhanov, A.I. Composite Type Equations and Inverse Problems; VSP: Utrecht, The Netherlands, 1999.

3. Lorenzi, A. Introduction to Identification Problems via Functional Analysis; VSP: Utrecht, The Netherlands, 2001.

4. Anikonov, Y.E. Inverse Problems for Kinetic and Other Evolution Equation; VSP: Utrecht, The Netherlands, 2001.

5. Belov, Y.Y. Inverse Problems for Partial Differntial Equations; VSP: Utrecht, The Netherlands, 2002.

6. Lavrentiev, M.M. Inverse Problems of Mathematical Physics; VSP: Utrecht, The Netherlands, 2003.

7. Isakov, V. Inverse Problem for Partial Differential Equations; Springer: New York, NY, USA, 2009.

8. Kabanikhin, S.I. Inverse and Ill-Posed Problems: Theory and Applications; Walter de Gruyter: Berlin, Germany, 2012.

9. Hasanov, H.A.; Romanov, V.G. Introduction to Inverse Problems for Differential Equations; Springer: New York, NY, USA, 2017.

10. Cannon, J.R.; Lin, Y. Determination of a Parameter $p(t)$ in some Quasi-Linear Parabolic Differential Equations. Inverse Probl. 1988, 4, 35-45. [CrossRef]

11. Ivanchov, M. Inverse Problems for Equations of Parabolic Type; WNTL Publishers: Lviv, Ukraine, 2003.

12. Valitov, I.R.; Kozhanov, A.I. Inverse Problems for Hyperbolic Equations: The Case of Time-Dependent Unknown Coefficients. Vestn. Novosib. Gos. Univ. Ser. Mat. Mekh. Inform. 2006, 6, 3-18. (In Russian)

13. Safiullova, R.R. Inverse Problem for a Second Order Hyperbolic Equation with an Unknown Time-Dependent Coefficient. Vestnik YuUrGU. Ser. Mat. Model. Progr. 2013, 6, 73-86. (In Russian)

14. Pyatkov, S.G.; Safonov, E.I. On Some Classes of Linear Inverse Problems for Parabolic Systems of Equations. Sib. Èlektron. Mat. Izv. 2014, 11, 777-799. (In Russian)

15. Slodic̈ka, M. Determination of a Solely Time-Dependent Source in a Semilinear Parabolic Problem by Means of Boundary Measurements. J. Comput. Appl. Math. 2015, 289, 433-440.

16. Kozhanov, A.I. Parabolic Equation with Unknown Time-Dependent Coefficients. Comput. Math. Math. Phys. 2017, 57, 956-966. [CrossRef]

17. Barbu, V.; Marinoschi, G. An Identification Problem for a Linear Evolution Equation in a Banach Space. Discret. Contin. Dyn. S 2020, 13, 1429-1440. [CrossRef]

18. Kozhanov, A.I. Questions of Posing and Solvability of Linear Inverse Problems for Elliptic Equations. J. Inverse Ill-Posed Probl. 1997, 5, 337-352. [CrossRef]

19. Frolenkov, I.V.; Kriger, E.N. Identification Problem of Coefficient in the Special Form at Source Function for Multi-Dimensional Parabolic Equation with Cauchy Data. J. Sib. Fed. Univ. Math. Phys. 2013, 6, 186-199.

20. Kriger, E.N.; Frolenkov, I.V. An Identification Problem of Nonlinear Lowest Term Coefficient in the Special Form for Two-Dimensional Semilinear Parabolic Equation. J. Sib. Fed. Univ. Math. Phys. 2016, 9, $180-191$. [CrossRef]

21. Lorenzi, A. Recovering Two Constans in a Linear Parabolic Equation. Inverse Problems in Applied Sciences. J. Phys. Conf. Ser. 2007, 73, 012014. [CrossRef]

22. Lorenzi, A.; Mola, G. Identification of a Real Constant in Linear Evolution Equation in a Hilbert Spaces. Inverse Probl. Imaging 2011, 5, 695-714. [CrossRef]

23. Mola, G. Identification of the Diffusion Coefficient in Linear Evolution Equation in a Hilbert Spaces. J. Abstr. Differ. Appl. 2011, 2, 18-28.

24. Lorenzi, A.; Mola, G. Recovering the Reaction and the Diffusion Coefficients in a Linear Parabolic Equations. Inverse Probl. 2012, 28, 075006. [CrossRef]

25. Mola, G.; Okazawa, N.; Prüss, J.; Yokota, T. Semigroup-Theoretic Approach to Identification of Linear Diffusion Coefficient. Discret. Contin. Dyn. Syst. S 2016, 9, 777-790.

26. Kozhanov, A.I. Inverse Problems of Finding of Absorption Parameter in the Diffusion Equation. Math. Notes 2019, 106, 378-389. [CrossRef] 
27. Kozhanov, A.I. The Heat Transfer Equation with an Unknown Heat Capacity Coefficient. J. Appl. Ind. Math. 2020, 14, 104-114. [CrossRef]

28. Lyubanova, A.S. Identification of a Constant Coefficient in an Elliptic Equation. Appl. Anal. 2008, 87, 1121-1128. [CrossRef]

29. Kozhanov, A.I.; Safiullova, R.R. Determination of Parameter in Telegraph Equation. Ufa Math. J. 2017, 9 , 62-75. [CrossRef]

30. Courant, R. Partial Differential Equations; Interscience: New York, NY, USA; London, UK, 1962.

31. Sobolev, S.L. Some Applications of Functional Analysis in Mathematical Physics; American Mathematical Society: Providence, RI, USA, 1991.

32. Triebel, H. Interpolation Theory, Function Spaces, Differential Operators; North Holland Publ.: Amsterdam, The Netherlands, 1978.

33. Dzhenaliev M.T. On the Theory of Linear Boundary Value Problems for Loaded Differential Equations; Institute of Theoretical and Applied Mathematics: Almaty, Kazakhstan, 1995. (In Russian)

34. Nakhushev A.M. Loaded Equations and Their Applications; Nauka: Moscow, Russia, 2012. (In Russian)

35. Aviles P.; Sandefur J. Nonlinear Second Order Equations with Applications to Partial Differential Equations. J. Differ. Equ. 1985, 58, 404-427. [CrossRef]

36. Yakubov, S.Y. Linear Differential-Operator Equations and Their Applications; ELM: Baku, Azerbaijan, 1985. (In Russian)

37. Lions J.L. Quelques Methods de Resolution des Problems aux Limites non Lineares; Dunod Cauthier-Villars: Paris, France, 1969.

38. Ladyzhenskaya O.A.; Uraltseva N.N. Linear and Quasilinear Elliptic Equations; Academic Press: New York, NY, USA; London, UK, 1987.

39. Scapellato, A. New perspectives in the theory of some function spaces and their applications. In Proceedings of the AIP Conference Proceedings, Thessaloniki, Greece, 25-30 September 2017; Volume 1978, p. 140002.

(C) 2020 by the authors. Licensee MDPI, Basel, Switzerland. This article is an open access article distributed under the terms and conditions of the Creative Commons Attribution (CC BY) license (http:/ / creativecommons.org/licenses/by/4.0/). 